\title{
Increased ATP synthesis might counteract hepatic lipid accumulation in acromegaly
}

Paul Fellinger, ${ }^{1}$ Peter Wolf, ${ }^{1}$ Lorenz Pfleger, ${ }^{1,2}$ Patrik Krumpolec, ${ }^{2}$ Martin Krssak, ${ }^{1,2}$ Kristaps Klavins, ${ }^{3}$ Stefan Wolfsberger, ${ }^{4}$ Alexander Micko, ${ }^{4}$ Patricia Carey, ${ }^{3}$ Bettina Gürtl, ${ }^{3}$ Greisa Vila, ${ }^{1}$ Wolfgang Raber, ${ }^{1}$ Clemens Fürnsinn, ${ }^{1}$ Thomas Scherer, ${ }^{1}$ Siegfried Trattnig, ${ }^{2}$ Alexandra Kautzky-Willer, ${ }^{1}$ Michael Krebs, ${ }^{1}$ and Yvonne Winhofer ${ }^{1}$

'Division of Endocrinology and Metabolism, Department of Medicine III, and ' 2 Centre of Excellence - High Field MR Centre, Department of Biomedical Imaging and Image-guided Therapy, Medical University of Vienna, Vienna, Austria. ${ }^{3}$ CeMM Research Center for Molecular Medicine of the Austrian Academy of Sciences, Vienna, Austria. ${ }^{4}$ Department of Neurosurgery, Medical University of Vienna, Vienna, Austria.

Patients with active acromegaly (ACRO) exhibit low hepatocellular lipids (HCL), despite pronounced insulin resistance (IR). This contrasts the strong association of IR with nonalcoholic fatty liver disease in the general population. Since low HCL levels in ACRO might be caused by changes in oxidative substrate metabolism, we investigated mitochondrial activity and plasma metabolomics/ lipidomics in active ACRO. Fifteen subjects with ACRO and seventeen healthy controls, matched for age, BMI, sex, and body composition, underwent ${ }^{31} \mathrm{P} /{ }^{1} \mathrm{H}-7-\mathrm{T}$ MR spectroscopy of the liver and skeletal muscle as well as plasma metabolomic profiling and an oral glucose tolerance test. Subjects with ACRO showed significantly lower HCL levels, but the ATP synthesis rate was significantly increased compared with that in controls. Furthermore, a decreased ratio of unsaturated-tosaturated intrahepatocellular fatty acids was found in subjects with ACRO. Within assessed plasma lipids, lipidomics, and metabolomics, decreased carnitine species also indicated increased mitochondrial activity. We therefore concluded that excess of growth hormone (GH) in humans counteracts HCL accumulation by increased hepatic ATP synthesis. This was accompanied by a decreased ratio of unsaturated-to-saturated lipids in hepatocytes and by a metabolomic profile, reflecting the increase in mitochondrial activity. Thus, these findings help to better understanding of $\mathrm{CH}$-regulated antisteatotic pathways and provide a better insight into potentially novel therapeutic targets for treating NAFLD.

Conflict of interest: The authors have declared that no conflict of interest exists.

Copyright: ( 2020 , American Society for Clinical Investigation.

Submitted: October 30, 2019

Accepted: February 12, 2020

Published: March 12, 2020.

Reference information: /CI Insight. 2020;5(5):e134638

https://doi.org/10.1172/jci. insight.134638.

\section{Introduction}

Acromegaly (ACRO) is characterized by a unique state of insulin resistance (IR), presumably due to the metabolic effects of increased growth hormone $(\mathrm{GH})$ concentrations, which is associated with increased lipolysis and reduced total body fat. Recent studies have shown that hepatocellular lipid (HCL) content especially is significantly reduced in ACRO (1-3), which stands in contrast to ectopic lipid accumulation and nonalcoholic fatty liver disease (NAFLD) as prevalent attributes of IR in the general population (4-6). In murine models, supplementation of $\mathrm{GH}$ and insulin-like growth factor 1 (IGF1) improved mitochondrial function and reduced oxidative stress, de novo lipogenesis, and hepatic lipid uptake (7-9). In healthy human subjects, it was shown that GH infusion resulted in an increased muscular mitochondrial oxidative capacity (10).

However, the potential mechanisms linking GH action with low hepatic lipid contents are still unclear. A recent review suggests that GH or IGF1 might be applicable for the treatment of steatohepatitis or cirrhosis (9). A trial investigating the effectiveness of GH treatment in reducing HCL levels in patients with NAFLD is currently underway (NCT02217345), and a very recent study investigating tesamorelin, a GH-releasing hormone analog that restores endogenous pulsatile GH secretion, showed a significant reduction of HCL levels as well as a reduced progression of fibrosis in HIV-positive patients with NAFLD (11). Therefore, a better understanding of the molecular mechanisms underlying low HCL levels in ACRO could help identify GH-regulated pathways providing the chance for discovering novel therapeutic targets for treating NAFLD. We hypothesized that increased mitochondrial 
Table 1. Baseline characteristics of subjects with ACRO and controls

\begin{tabular}{|c|c|c|c|}
\hline & ACRO & Controls & $P$ value \\
\hline$n$ & 15 & 17 & NS \\
\hline $\operatorname{Sex}(F / M)$ & $6 / 9$ & $8 / 9$ & NS \\
\hline Age & $48.0(12.3)$ & $44.8(13.0)$ & NS \\
\hline BMI & $26.6(5.1)$ & $26.8(5)$ & NS \\
\hline Fat-free mass (\%) & $75.3(10.5)$ & $72.1(9)$ & NS \\
\hline Fat mass (kg) & $21.1(15.2)$ & $21.7(8.5)$ & NS \\
\hline HbA1c (\%) & $5.5(0.5)$ & $5.2(0.3)$ & NS \\
\hline RR systolic (mmHg) & 144.8 (20.9) & $122.8(15.2)$ & $<0.01$ \\
\hline RR diastolic (mmHg) & $87.9(14)$ & $79.3(14.2)$ & NS \\
\hline IGF1 (ng/ml) & $715.2(253.4)$ & $178.1(29.5)$ & $<0.001$ \\
\hline Triglycerides (mg/dl) & $119.1(65)$ & $124.9(88.1)$ & NS \\
\hline HDL cholesterol (mg/dl) & $54.6(13.6)$ & $51.1(8.3)$ & NS \\
\hline LDL cholesterol (mg/dl) & $113.9(37.4)$ & $108.7(32.5)$ & NS \\
\hline GOT (U/I) & $20.2(5.1)$ & $23.1(10.6)$ & NS \\
\hline GPT (U/I) & $20.7(9.3)$ & $24.2(10.1)$ & NS \\
\hline$\gamma-\mathrm{GT}(\mathrm{U} / \mathrm{I})$ & $26.5(43.7)$ & $23(13.1)$ & NS \\
\hline Fasting glucose (mg/dl) & $97.1(15.1)$ & $85.8(8.8)$ & 0.01 \\
\hline Fasting insulin (ng/ml) & $14.5(7.3)$ & $10.1(6.7)$ & NS \\
\hline Fasting C-peptide (ng/ml) & $3.3(0.96)$ & $2.2(0.79)$ & 0.002 \\
\hline OGIS & $556.7(276.0)$ & $492.3(223.4)$ & NS \\
\hline$\%$ HOMA2-B & $126.5(45.1)$ & $118.0(34.9)$ & NS \\
\hline HOMA2-S & $7.88(35.5)$ & $104.32(51.0)$ & 0.03 \\
\hline HOMA2-IR & $1.7(1.0,2.5)$ & $1.0(0.8,1.4)$ & 0.04 \\
\hline Matsuda index & $3.6(0.9)$ & $5.6(0.9)$ & NS \\
\hline \multicolumn{4}{|c|}{$\begin{array}{l}\text { Mean (SD). F/M, female/male; IGF1, insulin-like growth factor 1; RR, blood pressure after Riva-Rocci; HOMA, } \\
\text { homeostasis model; OGIS, oral glucose index sensitivity. }\end{array}$} \\
\hline
\end{tabular}

activity in the livers of patients with ACRO might be causally linked with the observed low ectopic lipid content. Noninvasive metabolic magnetic resonance spectroscopy (MRS) of the liver and skeletal muscle in combination with metabolomic analyses of plasma was used to investigate the consequences of $\mathrm{GH}$ excess.

\section{Results}

\section{Comparison between subjects with ACRO and controls}

Subjects with ACRO and controls were of comparable age, BMI, and fat-free mass (FFM). In subjects with ACRO, systolic blood pressure was significantly higher and fasting insulin sensitivity was lower. Dynamic insulin sensitivity was not significantly different between the groups, even though Matsuda index tended to be lower in subjects with ACRO (Table 1). None of the subjects with ACRO were on somatostatin analogs therapy.

\section{Hepatic energy and lipid metabolism}

Liver measurements were successfully obtained from all but 1 patient with ACRO, who did not tolerate lying in the lateral position, and $2{ }^{31} \mathrm{P}-\mathrm{MRS}$ examinations were excluded due to weak phosphorus spectra. HCL levels were significantly lower in subjects with ACRO $(n=12)$ compared with controls $(n=$ 17), as depicted in Figure 1A.

The proportion of unsaturated lipids to total HCL was significantly different between the groups, with subjects with ACRO showing lower unsaturation index (UI) compared with controls (Figure 1B).

The ATP synthesis rate, assessed by hepatic mitochondrial ATP synthase activity ( $\mathrm{h} \_\mathrm{k}_{\text {ATP }}$ ), adjusted to log-transformed HCL was significantly higher in subjects with ACRO compared with controls (Figure 1C). 
A

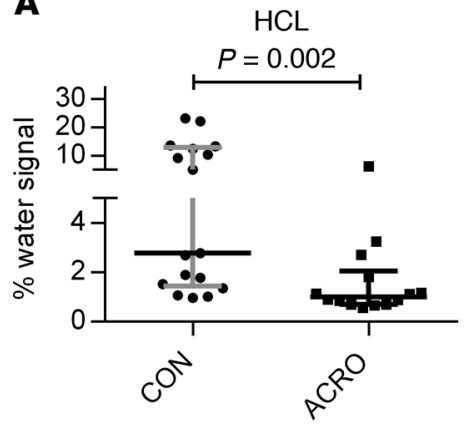

B

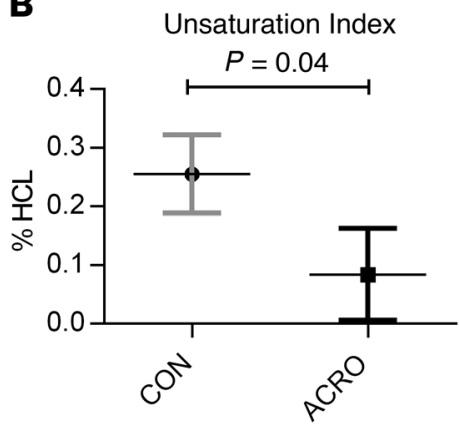

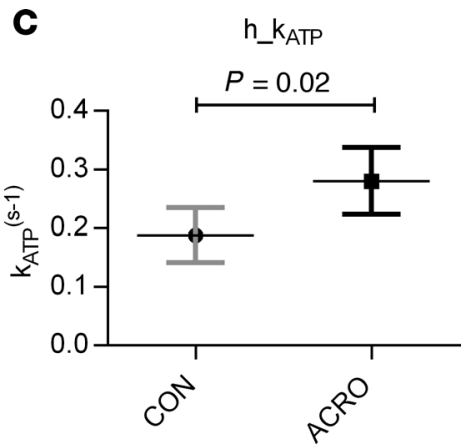

Figure 1. 7-T MR spectroscopy of the liver. 7-Tesla MR examinations in patients with active acromegaly (ACRO, $n=12$ ) and healthy controls (CON, $n=17$ ) showed (A) significantly decreased HCL (ACRO 1.0\% [0.7\%-1.8\%] vs. CON 2.8\% [1.5\%-12.4\%]) using Mann-Whitney U test (median \pm IQR); (B) a lower unsaturation index in the liver (ACRO $8.4 \%$ [0.6\%-16.3\%] vs. CON 25.5\% [18.9\%-32.2\%]) using a multivariable model adjusting for HCL (adjusted mean \pm $95 \% \mathrm{Cl}$ ); and (C) significantly increased hepatic ATP synthase activity (ACRO (0.28 s $\mathrm{s}^{-1}$ [0.22-0.34 $\left.\mathrm{s}^{-1}\right]$ vs. CON $0.19 \mathrm{~s}^{-1}$ [0.14-0.24 s-1]), using a multivariable model adjusting for $\mathrm{HCL}$ (adjusted mean $\pm 95 \% \mathrm{Cl}$ ). $\mathrm{HCL}$, hepatocellular lipid content.

\section{Skeletal muscle energy and lipid metabolism}

Valid spectra of intramyocellular metabolites were obtained from 7 subjects with ACRO and 12 controls. There was no significant difference regarding BMI, age, sex, and FFM between the groups. Intramyocellular lipid content (IMCL) was significantly lower in subjects with ACRO compared with controls (Figure $2 A)$. Adjusted for IMCL, ATP synthase activity $\left(\mathrm{m}_{-} \mathrm{k}_{\text {ATP }}\right)$ in the muscle was significantly lower in subjects with ACRO compared with controls (Figure $2 \mathrm{~B}$ ). Phosphocreatinine kinase activity $\left(\mathrm{k}_{\mathrm{PCr}}\right)$ as adjusted for IMCL was not significantly different between subjects with ACRO and controls (Figure 2C).

\section{Hepatic phosphor metabolites}

Absolute quantification of hepatic phosphor metabolites in the MR spectra was obtainable in 6 subjects with ACRO and 15 controls and showed significantly elevated high energy metabolites, such as $\gamma$-ATP and $\alpha$-ATP, in subjects with ACRO compared with controls (Table 2). Other high-energy phosphometabolites, NADH and UDPG, were also higher in subjects with ACRO, but differences were not significant.

Subjects with ACRO showed a significantly elevated concentration of hepatic phosphodiester (PDE), which is indicative of cell membrane breakdown. This difference was mainly driven by a difference in the PDE compound glycerophosphoethanolamine (GPE), while glycerophosphocholine (GPC) only tended to be higher in subjects with ACRO. Phosphomonoester (PME), a marker for cell membrane turnover, also tended to be elevated in subjects with ACRO, the difference was, however, statistically not significant. While hepatic phosphoethanolamine (PE) was significantly higher in subjects with ACRO, phosphocholine (PC) was not significantly affected. Furthermore, neither the ratio of PME

A

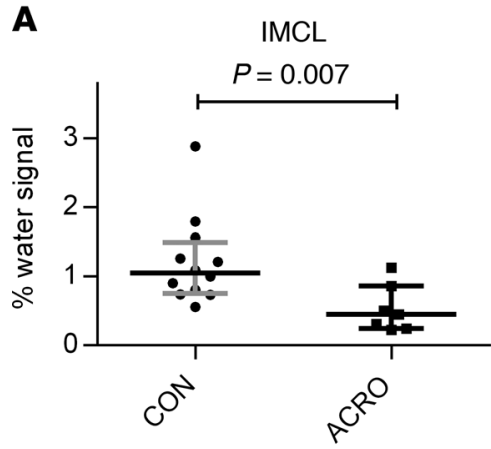

B

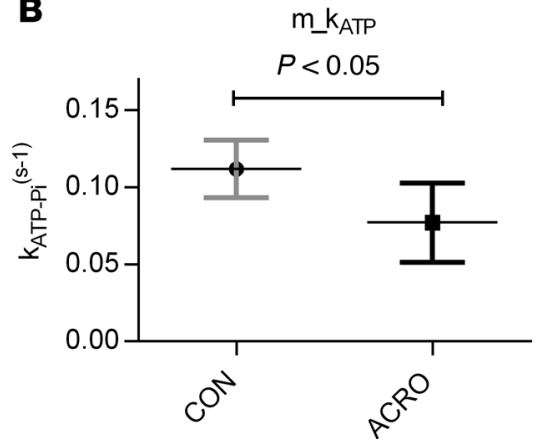

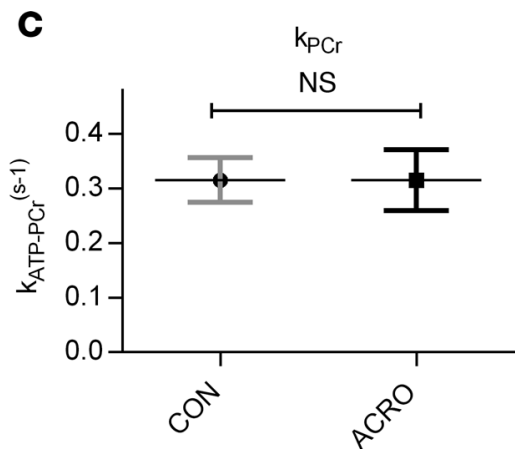

Figure 2. 18. 7-T MR spectroscopy of skeletal muscle. 7-Tesla MR examinations in patients with active acromegaly (ACRO, $n=7)$ and healthy controls (CON, $n=12$ ) showed $(\mathbf{A})$ decreased IMCL (0.45\% [0.25\%-0.86\%] vs. 1.05\% [0.76\%-1.49\%]) using Mann-Whitney $U$ test (median \pm IQR); (B) a lower muscular ATP synthase activity (0.08 s-1 [0.05-0.1 s-1] vs. $0.11 \mathrm{~s}^{-1}\left[0.09-0.13 \mathrm{~s}^{-1}\right]$ ) using a multivariable model adjusting for IMCL (adjusted mean $\pm 95 \% \mathrm{Cl}$ ); and (C) no difference in phosphocreatinine kinase (ACRO $0.32 \mathrm{~s}^{-1}\left[0.27-0.36 \mathrm{~s}^{-1}\right] \mathrm{vs}$. CON $0.32 \mathrm{~s}^{-1}\left[0.26-0.37 \mathrm{~s}^{-1}\right]$ ) activity using a multivariable model adjusting for IMCL (adjusted mean $\pm 95 \% \mathrm{Cl}$ ). IMCL, intramyocellular lipid content. 
Table 2. Differences in phosphometabolites using MRS

\begin{tabular}{lccc}
\hline Metabolites & ACRO (mM) & Controls (mM) & P value \\
$\gamma$-ATP & $2.98(0.5)$ & $2.49(0.4)$ & 0.029 \\
$\alpha$-ATP & $2.8(0.41)$ & $2.37(0.4)$ & 0.042 \\
NADH & $1.36(0.47)$ & $1.11(0.27)$ & NS \\
UDPG & $1.11(0.31)$ & $0.84(0.3)$ & NS \\
Pi & $1.5(0.25)$ & $1.35(0.3)$ & NS \\
PME & $3.3(0.58)$ & $2.73(0.63)$ & NS \\
PE & $2.33(0.39)$ & $1.5(0.5)$ & 0.002 \\
PC & $0.97(0.34)$ & $1.23(0.25)$ & NS \\
PDE & $8.05(1.63)$ & $6.19(1.7)$ & 0.035 \\
GPC & $3.98(0.77)$ & $3.22(1.19)$ & NS \\
GPE & $4.07(1.01)$ & $2.97(0.8)$ & 0.016 \\
PME/PDE & $0.42(0.07)$ & $0.47(0.14)$ & NS \\
PtdCh & $0.39(0.13)$ & $0.3(0.12)$ & NS
\end{tabular}

Mean (SD). MRS, magnetic resonance spectroscopy; Pi, inorganic phosphate; PE, phosphoethanolamine; PC, phosphocholine; PME, phosphomonoester; GPE, glycerophosphoethanolamine; GPC, glycerophosphocholine; PDE, phosphodiester; PtdCh, phosphatidylcholine; ATP, adenosine triphosphate; UDPG, uridine diphosphoglucose.

to PDE nor the total numbers of other phosphor metabolites was significantly different between the 2 groups. The data on hepatic phosphor metabolites are summarized in Table 2.

\section{Metabolomics/lipidomics}

Metabolites. In total, 60 different metabolites were specified (Supplemental Figure 1A; supplemental material available online with this article; https://doi.org/10.1172/jci.insight.134638DS1). With regards to amino acids, there was a significant detectable difference in the concentration of serine, which was increased in subjects with ACRO compared with controls (Table 3). There were, however, no significant differences among the other analyzed detectable amino acids. A pathway analysis hinted at the potential difference between $\mathrm{ACRO}$ and controls regarding the glycine-serine-threonine pathway, but this was only a nonsignificant trend $(P=0.08)$. (Figure 3$)$ No significant fold change was observed for any of the other amino acids or pathways. Within the carnitine species, most species trended lower in subjects with ACRO, but only plasma butyrylcarnitine and hexanoylcarnitine were statistically significantly decreased in subjects with ACRO (Figure 4).

Lipids. In total, 260 lipid species were analyzed (Supplemental Figure 1B and Supplemental Figures 2-6). Total plasma free fatty acids (FFA) were not significantly different between ACRO and controls. Overall, only a few lipid species passed the prespecified threshold and were different between the 2 groups. Among the various FFA species, only C18:3 was lower in subjects with ACRO compared with controls (fold change, 0.69; $P<0.1$ ). Accordingly, levels of cholesteryl esters (CEs, 18:3) were also markedly lower in subjects with ACRO, whereas the other CE species did not differ between the 2 groups (Table 3 ).

Within PC lipids, increased levels of lysophosphatidylcholine (LPC) 18:0, as well as decreased phosphatidylcholine $\mathrm{PC}(36: 5)$ and ether phosphatidylcholine $\mathrm{PC}(\mathrm{O}-38: 6), \mathrm{PC}(\mathrm{O}-40: 7)$, and $\mathrm{PC}(\mathrm{O}-42: 5)$ levels, were found. There were no differences detected in the other PC O species. In the sphingomyelin class, SM d36:0 was found to be significantly lower in subjects with ACRO, while the levels of the others were comparable. In the lipid classes of ceramides, diacylglycerols, triacylglycerols, phosphatidylethanolamines (LPE, PE, PE-O), phosphatidylglycerol (PG), phosphatidylinositol (PI), and phosphatidylserine (PS) no significant differences were detectable. Fasting plasma glycerol levels were also not significantly different between the subjects with ACRO and controls ( $0.08 \pm 0.01$ vs. $0.09 \pm 0.03 \mu \mathrm{g} / \mu \mathrm{l}, P=\mathrm{NS})$.

\section{Discussion}

This study demonstrates for the first time to our knowledge that low HCL levels in a state of GH excess is associated with increased hepatic ATP synthesis. Furthermore, the ratio of unsaturated-to-saturated intracellular FA was decreased in subjects with ACRO. IMCL was also reduced in subjects with ACRO, but in contrast to that in the liver, ATP synthase activity was decreased rather than elevated in skeletal muscle. 
Table 3. Differences in metabolomics between subjects with ACRO and controls passing the prespecified thresholds of the volcano plot

\begin{tabular}{|c|c|c|c|c|}
\hline Species & FC & $\log _{2}(F C)$ & $-\log _{10}(P)$ & $P$ value \\
\hline Butyrylcarnitine & 0.76 & -0.4 & 1.63 & 0.02 \\
\hline PC 0-38:6 & 0.71 & -0.49 & 2.52 & $<0.01$ \\
\hline PC 0-40:7 & 0.7 & -0.52 & 2.04 & $<0.01$ \\
\hline PC 0-42:5 & 0.73 & -0.46 & 1.77 & 0.02 \\
\hline LPC 18:0 & 1.32 & 0.39 & 1.18 & 0.07 \\
\hline CE 18:3 & 0.67 & -0.59 & 1.1 & 0.08 \\
\hline C 18:3 & 0.69 & -0.54 & 1.01 & 0.098 \\
\hline
\end{tabular}

PC-0, ether phosphatidylcholines; PC, phosphocholine; SM, sphingomyelin; LPC, lysophosphatidylcholine; CE, cholesteryl ester.

In peripheral blood, metabolomic analyses showed no relevant changes in lipid species but revealed significantly decreased carnitine species in subjects with ACRO, further indicating increased mitochondrial activity.

Our data confirm previously published results that indicate that subjects with ACRO have significantly lower HCL levels than healthy controls $(1,2)$. In contrast to former studies, our controls were also matched for relative lean body mass in addition to being matched for sex, age, and BMI $(1,2)$. Median HCL levels in subjects with ACRO were $1.0 \%$ (ranging from $0.7 \%$ to $1.9 \%$ ) and therefore were comparable to previously published mean HCL values in active ACRO of $1.2 \%$ and $2 \%(1,2)$. Median HCL values in controls were also similar to those reported in previously published studies, where mean HCL values ranged from $2.4 \%$ to $6 \%,(1,12-14)$. Our results therefore confirm previous findings, that active ACRO is characterized by significantly reduced HCL, despite increased IR.

The potentially novel finding of this study is that low hepatic lipid content in subjects with ACRO was associated with an approximately 50\% higher hepatic ATP synthesis rate constant compared with controls. This leads us to conclude that low HCL levels are the consequence of increased mitochondrial activity.

As previously mentioned, hepatic ATP synthesis in the general population seems to increase directly in relation to HCL, followed by a decline of mitochondrial function, as seen in conditions, such as chronic IR, type 2 diabetes mellitus, and nonalcoholic steatohepatitis (NASH) (15). In general, patients with ACRO are known to have an accelerated oxidative metabolism, and previous studies have shown that administration of $\mathrm{GH}$ leads to enhanced expression of genes relevant to mitochondrial oxidation in healthy volunteers as well as in GH-deficient individuals $(10,16,17)$.

Transgenic mouse models could show that overexpression of GH leads to hepatomegaly and hepatocyte hypertrophy as well as an increased number of mitochondria in hepatocytes, with STAT5 and JAK2 being important regulators of these alterations $(18,19)$. However, hepatocellular size was not assessed by the currently applied methods.

As saturation transfer measurement of the Pi-to-ATP exchange rate includes the phosphorous cycling at the level of GAPDH and 3-phosphoglycerate kinase of the glycolytic and gluconeogenic pathways, the measured difference could also be due to increased hepatic gluconeogenesis in subjects with ACRO (4, 20, 21). Therefore, glycogen measurement in consequent studies will be of great interest. The analysis of peripheral acylcarnitine species, indicators for the efficiency of mitochondrial $\beta$ oxidation, revealed that subjects with ACRO have lower levels than controls. This indicates elevated $\beta$ oxidation under GH excess and hence underlines our findings from MRS measurements (22). A recently published phase II study about the effect of GS-0976, an inhibitor of hepatic ACC and potential drug against NAFLD, showed that peripheral acylcarnitine species decrease in responsive patients (23). Regarding serine, which we found increased in subjects with ACRO, a previous study showed that supplementation of serine in patients with NAFLD ameliorates hepatic steatosis (24). In contrast, patients with NAFLD have reduced plasma serine, while patients with NASH even suffer from severe serine deficiency (25). Through its role in glutathione synthesis, increased plasma serine, as we found in subjects with ACRO, could therefore help reduce oxidative stress and associated ROS, as caused by increased $\beta$ oxidation (25). 

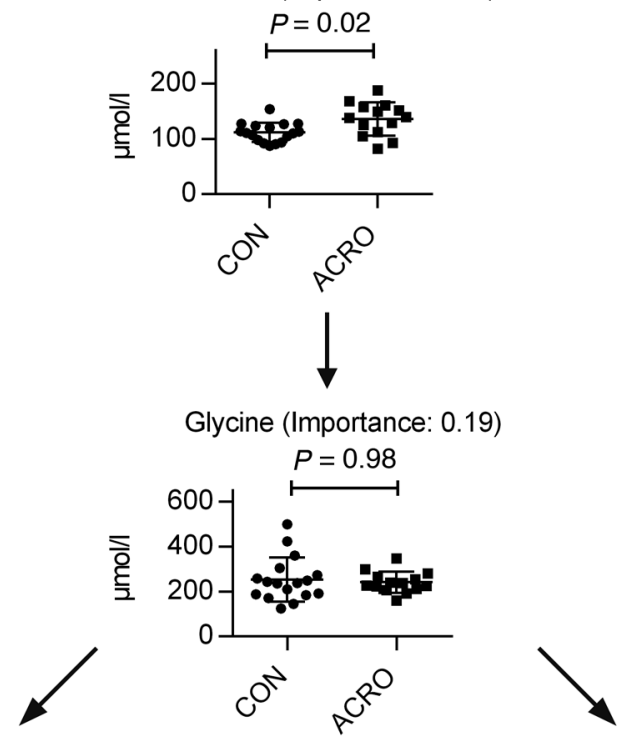

Serine (Importance: 0.14)

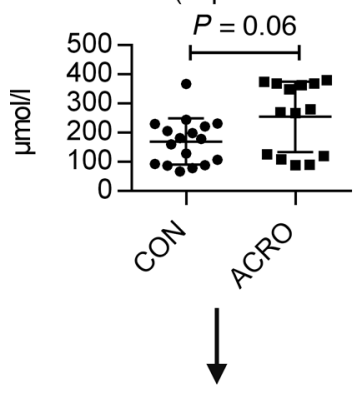

Sarcosine (Importance: 0.04)

Tryptophan (Importance: 0)

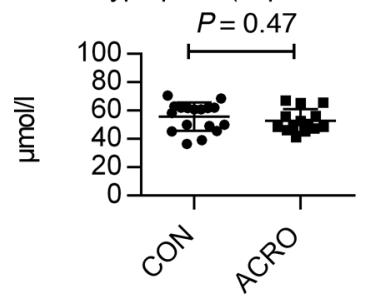

Figure 3. Kyoto Encyclopedia of Genes and Genomes pathway analysis of glycine, serine, and threonine metabolism, with measured parameters and their respective importance to the pathway and $\boldsymbol{P}$ values. Total matches, 5 of $48 . P=0.08$; mean \pm SD. Impact $=0.47012$. Metabolomics pathway data analysis was performed with MetaboAnalyst (https://www.metaboanalyst.ca).

In addition to low hepatic lipid content, we found a decreased UI, with the ratio of unsaturated lipids to the total lipid signal being approximately 3 times lower in subjects with ACRO compared with controls. In general, the relative amount of saturated lipids in the liver rises with elevated lipid content and decreased UI is found in NAFLD and NASH (26-28). Whether this is the consequence of unsaturated lipids being preferentially metabolized in the liver or due to different sources of lipids is unclear. However, altered lipid composition might reflect hepatic IR.

Further indicators for altered metabolic mechanisms in the livers of subjects with ACRO are that high energy phosphates, such as PME and PDE, which are considered to be markers for cell membrane synthesis and degradation, were elevated in subjects with ACRO (29-31). Our results might therefore support the hypothesis of increased hepatic metabolism and local inflammation in active ACRO.

Circulating FFA originating from adipose tissue as well as directly from nutrition are generally regarded as a major source for ectopic lipid accumulation, and there is evidence that aside from ectopic lipids and adipose tissue, GH-induced lipolysis plays a role in the interorgan transfer of lipids and the induction of IR (32, 33). Interestingly, no changes were found in concentrations of FFA species, except (C18:3), which was markedly lower in subjects with ACRO. In the CE species, 18:3 was also markedly lower in subjects with ACRO. Studies in the early 1990s, which showed that muscle glucose uptake and nonoxidative glucose metabolism 

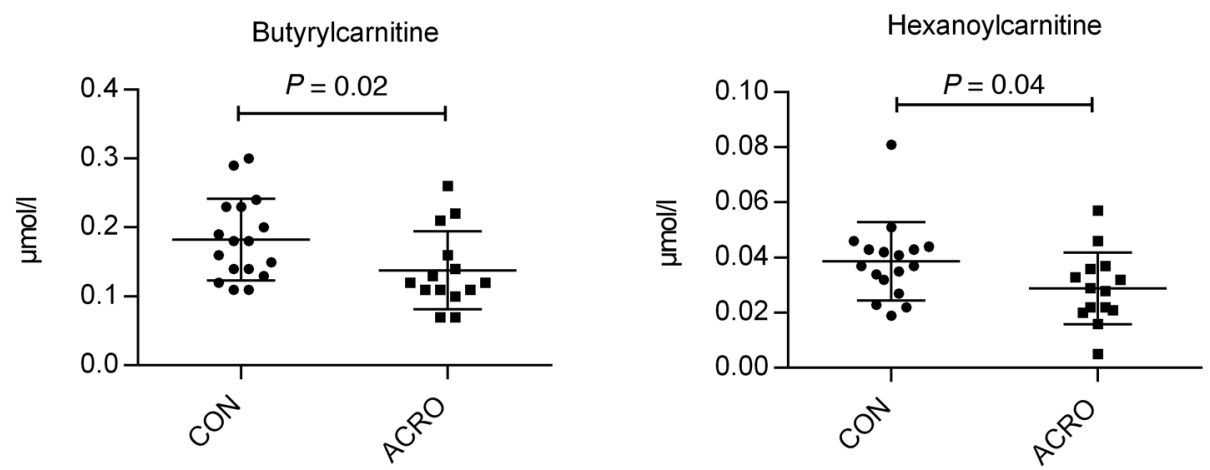

Figure 4. Significant changes to carnitine species comparing subjects with ACRO and controls. Butyrylcarnitine and hexanoylcarnitine (mean \pm SD). Metabolomics data analysis was performed with MetaboAnalyst (https://www.metaboanalyst.ca).

were reduced in subjects with ACRO, likewise failed to find differences in circulating FFA (16, 34). Since GH causes triglyceride lipolysis primarily in visceral adipose tissue, peripherally measured FFA concentrations do not necessarily represent circulating FFA in the portal vein and, therefore, lipid flux from white adipose tissue to the liver (9). The time of blood sampling could also be important, since a previous study showed that plasma FFA are significantly elevated in patients with active ACRO compared with patients treated during the night but not in the morning (35).

Like lipid deposition in the liver, intramyocellular lipid accumulation is also linked to peripheral insulin sensitivity in nondiabetic and prediabetic people (36-38). In contrast to previous studies where no change in IMCL was found in subjects with ACRO, our analysis showed significantly lower IMCL in subjects with ACRO compared with controls $(2,39)$. The application of ultra-high-field 7-Tesla MRS, with improved signal-to-noise ratio and calculation of IMCL in relation to total water signal rather than total phosphocreatine, could explain our finding. Contrary to our findings of increased ATP flux in the liver, $m \_k A T P ~-~ a s$ a parameter for muscular ATP synthase activity - was decreased in subjects with ACRO. To our knowledge, this is the first time that muscular ATP flux was assessed in active ACRO.

This result, however, contrasts with findings from healthy subjects, where GH infusion led to enhanced mitochondrial function, as assessed by muscle biopsy (10). Previous studies in patients with ACRO, however, showed that glucose uptake and glucose oxidation are reduced in peripheral muscle (16). As m_kATP assessed by MRS also represents nonoxidative glycolytic activity in the muscle, our readout might therefore not show mitochondrial activity directly but rather indicate a change to muscular metabolic substrate selections $(6,40)$. As only $\mathrm{m}_{-} \mathrm{kATP}$, but not $\mathrm{k}_{\mathrm{PCr}}$ was different between the 2 groups, this might also support this conclusion. Nevertheless, the difference could still be an indicator that resting muscular mitochondrial activity plays a role for IR in subjects with ACRO, as similar reductions were also found in insulin-resistant individuals $(6,40,41)$. Our contrary findings of increased ATP synthesis in the liver but reduced ATP synthesis in the muscle could possibly also be explained by increased gluconeogenesis in the liver in contrast to decreased glucose uptake and glucose oxidation in skeletal muscle, as previously reported (3).

The current study has some limitations that should be addressed. First, we were not able to analyze all MR spectra from ACRO due to poor signal quality, while repeated measurements were not possible, due to the patients undergoing transsphenoidal surgery immediately after the study day. Second, as we used a liquid chromatography-based method for the analysis of fatty acids it was not possible to differentiate between the different isomers. In addition, a second control group with NAFLD and IR would have been helpful to contrast all measurements done in this study; however, since this would have meant matching a very heterogeneous group with various parameters (measurement of IR and NAFLD), we decided to match for body composition.

In summary, nonfatty liver in ACRO is associated with increased hepatic mitochondrial activity and with a decreased ratio of unsaturated-to-total intracellular lipids. Decreased plasma carnitine species in subjects with ACRO support this finding. IMCL was also decreased in subjects with ACRO, but ATP flux (indicative of muscular ATP synthase activity) was also decreased, which contrasts the finding in the liver. Thus, we conclude that GH excess is associated with mitochondrial changes that might lead 
A

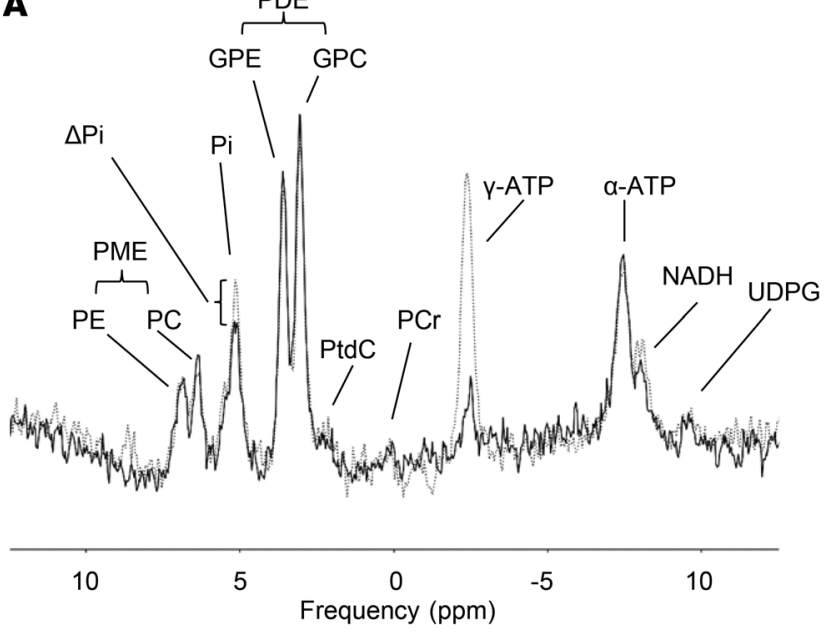

B

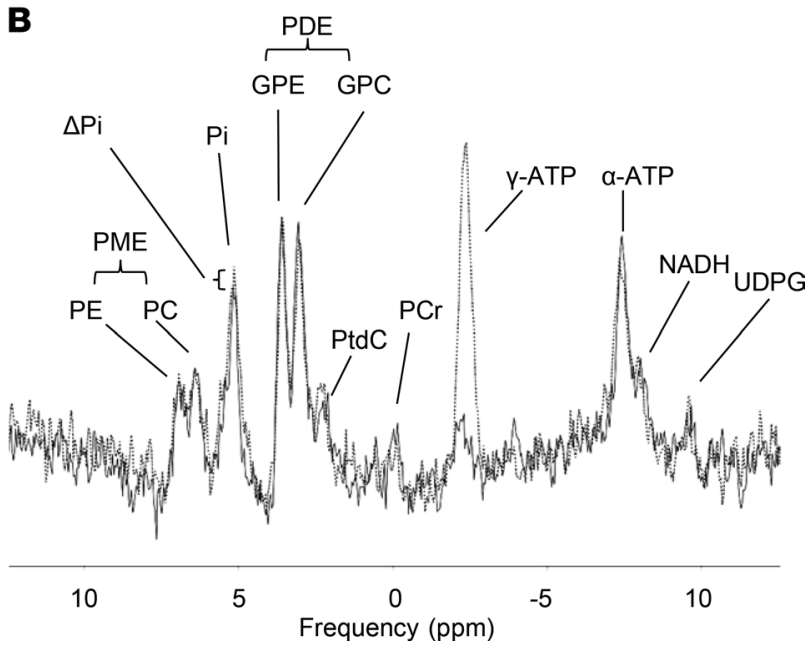

Figure 5. ${ }^{31}$ P MRS procedure of individuals with similar hepatic lipid content (1.1\% vs. 1.8\%). (A) A patient with acromegaly and (B) a matched control. The dotted line represents the normal spectrum while the continuous line represents the spectrum after applying a saturation pulse on $\gamma$-ATP. Difference in $\mathrm{Pi}(\triangle \mathrm{Pi})$ was used to calculate activity of ATP synthase (h_k_ATP). All amplitudes are in arbitrary units. PE, phosphoethanolamine; PC, phosphocholine; $\mathrm{Pi}$, inorganic phosphate; PME, phosphomonoester; GPE, glycerophosphoethanolamine, GPC, glycerophosphocholine; PDE, phosphodiester; PtdCh, phosphatidylcholine; PCr, phosphocreatine; UDPG, uridine diphosphoglucose; MRS, magnetic resonance spectroscopy.

to peripheral IR but, at the same time, prevent hepatic steatosis and liver dysfunction. Hence, therapies targeting these pathways might be a future option for treating NAFLD.

\section{Methods}

Subjects. Fifteen subjects with active ACRO and seventeen healthy controls, matched for age, sex, BMI, and FFM, were included in the study. Diagnosis of ACRO was confirmed by elevated serum IGF1 concentrations and lack of GH level suppression ( $>1 \mathrm{ng} / \mathrm{ml}$ ) after ingestion of $75 \mathrm{mg}$ glucose during an oral glucose tolerance test. Twenty-seven healthy subjects, who were recruited via a public display for the study, were invited to a screening visit to assess body weight, height, and body composition via bioelectrical impedance analysis (BIA) using Nutriguard M (Data Input), and seventeen suitable healthy subjects were then selected to match the patients with ACRO.

The sample size was chosen after performing a sample size calculation based on previously published data, given that ACRO is a rare disease.

All subjects met the inclusion criteria of being between 18 and 75 years of age. Exclusion criteria consisted of standard MR contraindications (claustrophobia, metal devices in or on the subject's body), severe liver or kidney disorder, any acute inflammatory disease within 2 weeks prior to the study, pregnancy, and nursing. Prior to the study day, all healthy controls were screened for any acute or chronic metabolic or cardiac diseases, and the intake of medication affecting glucose or lipid metabolism was ruled out. Anthropometric data were recorded, including FFM assessed using BIA to ensure good matching.

Experimental protocol. Examinations were performed under resting conditions in the morning after an overnight fast of at least 8 hours, and participants were asked to refrain from physical training and to ingest an isocaloric diet for 3 days prior to the day of the study. In women of childbearing age, a pregnancy test was performed prior to any study-related activity.

MRS. ${ }^{31} \mathrm{P} /{ }^{1} \mathrm{H}$ MRS of liver and muscle was performed on a 7.0-T whole-body MR system (Magnetom, Siemens) using 1 of 2 double-tuned $\left({ }^{31} \mathrm{P} /{ }^{1} \mathrm{H}\right)$ surface coils (both Rapid Biomedical Ltd.), with diameters of $10 \mathrm{~cm}$ and $14 \mathrm{~cm}\left({ }^{31} \mathrm{P}\right.$ channel). The liver measurement was conducted with participants lying in a lateral position with the right lobe of the liver positioned above the $10-\mathrm{cm}$ coil. In cases where the desired positioning could not be achieved due to the relatively small-bore diameter, the volunteers were measured in supine position using the bigger coil strapped down on their chest so that the liver was centered below the coil. The exact measurement position was adjusted to localizer images. Hepatic lipid content was measured using localized single voxel GUSTEAU sequence for evaluation of individual spin-spin relaxation times (T2) and then calculated from the ratio of summed area of fat to that of water plus fat, with all signals corrected for both T1 and T2 according to a previous published protocol (26). For analysis of hepatic 
lipid composition, lipid UI was calculated as a ratio between the signal of unsaturated lipids to total lipid signal. The estimation of $\mathrm{h}_{-} \mathrm{k}_{\mathrm{ATP}}$ by ${ }^{31} \mathrm{P}$ saturation transfer was performed as previously described (20). Examples of a patient's as well as a control's MRS can be seen in Figure 5. The 3D clinical shift imagingbased method for absolute quantification was used for assessing concentrations of phosphor-containing hepatic metabolites using the phantom replacement method as previously described (42).

For the skeletal muscle measurements, participants were asked to lie in a supine position with the calf muscle positioned over $10-\mathrm{cm}$ diameter surface coil. IMCL was assessed in the soleus muscle with 2 localized single-voxel ${ }^{1} \mathrm{H}$ MRS sequences, after adjusting the RF power for maximizing the receive signal and calculating the ratios of area of IMCL methylene resonance to that of water, following the individual relaxation correction as a percentage of total tissue water MRS signal (43). The measurement of the unidirectional forward rates of oxidative $\mathrm{m} \_\mathrm{k}_{\mathrm{ATP}}$ as well as $\mathrm{k}_{\mathrm{PCr}}$ was carried out using similar settings as previously described (20).

Glucose tolerance and metabolic parameters. Following the MRS measurement, blood samples were taken for assessing fasting concentrations of glucose, insulin, C-peptide, GH, and IGF1 as well as of parameters relevant to the function of liver, kidney, thyroid, pituitary, and parathyroid. In addition, a $75 \mathrm{~g}$ 2-hour oral glucose tolerance test was performed, and GH, glucose, insulin, and C-peptide concentrations were measured at 30, 60, 90, and 120 minutes. Glucose, insulin, and C-peptide values were used for calculating parameters of insulin sensitivity and $\beta$ cell function. HOMA2 models for $\beta$ cell function, $\beta$ cell sensitivity, and IR were calculated for characterizing the fasting state, while the oral glucose insulin sensitivity index and Matsuda index were used for evaluating dynamic insulin sensitivity (44-46). Unless stated otherwise, all parameters were measured by routine lab methods at the Department of Laboratory Medicine of the Medical University of Vienna (http://www.kimcl.at).

FFA/metabolomic/lipidomic analysis. Free glycerol in plasma was measured using a colorimetric triglyceride determination kit (F6428, MilliporeSigma) according to the manufacturer's instructions. The absorbance was read at a 540-nm wavelength with a multimode microplate reader (Synergy H1M, Fa. BioTek instruments Inc.).

For quantitative analysis of FFA in plasma samples, the derivatization procedure followed by liquid chromatography-mass spectrometry (LC-MS) analysis was used. Chromatographic separation was carried out on an ultra-high-performance liquid chromatography (UHPLC) system (Vanquish, Thermo Scientific) equipped with a reversed phase-based column. The Orbitrap based mass spectrometer (Orbitrap Fusion Lumos, Thermo Scientific) operating in full MS scan mode was used for the detection of derivatized fatty acids. A 10-point linear calibration curve with internal standardization was constructed for the quantification of the metabolites.

The semiquantitative lipid analysis was performed by LC-MS as previously described (47). The chromatographic separation of lipids was carried out on the above-mentioned UHPLC system equipped with a reversed phase-based analytical column. Lipid detection was performed using the previously mentioned Orbitrap-based mass spectrometer operating in full MS scan mode in both ESI-positive and -negative ionization mode. Collision-induced dissociation and higher energy collisional dissociation were used to obtain MS2 spectra. A more detailed methodological description is provided in the Supplemental Methods.

The targeted quantitative metabolite analysis was performed by LC-MS. The metabolite separation was carried out using a hydrophilic interaction chromatography-based analytical column. Metabolites of interest were detected using an Orbitrap Q Exactive (Thermo Scientific) mass spectrometer operating in a full MS scan mode. Seven-point linear calibration curves with internal standardization were constructed for the quantification of the metabolites. All data processing was performed with the TraceFinder 4.1 software (Thermo Scientific).

Statistics. Measurements were checked for normal distribution by visual methods and were further log transformed if necessary. Where not stated otherwise, data are presented as mean $\pm \mathrm{SD}$, median and interquartile range, or percentage. Normally distributed data were compared using unpaired 2-tailed Student's $t$ test, and nonnormally distributed data were compared using the nonparametric Mann-Whitney $U$ test and a multivariable model, adjusting for lipid content of liver or muscle, respectively. As previous studies showed that parameters of mitochondrial ATP turnover as well as the ratio of saturated/unsaturated lipids correlated directly with lipid content, we adjusted these parameters to the lipid content. Correlation analysis was performed via Pearson's correlation coefficient. Statistical significance was set at $\alpha<0.05$. Metabolomic and lipidomic data analysis was performed using MetaboAnalyst (https://www.metaboanalyst.ca) (48). 
Before analysis, data were log transformed and Pareto scaling was applied. Threshold values for volcano plot were 1.3 for fold change and a $P$ value of 0.1 . All other statistical analysis was performed using SAS enterprise, and graphs were created using GraphPad Prism. $P$ values of less than 0.05 were considered significant.

Study approval. Written informed consent was received from all participants prior to inclusion in the study. The protocol was approved by the Human Ethics Committee of the Medical University of Vienna (ethics committee no., 2039/2013). The study was reported under clinical trial no. NCT02115906.

\section{Author contributions}

PF, PW, LP, M. Krssak, PK, KK, PC, BG, GV, CF, M. Krebs, and YW designed the study and performed the experiments. PF, YW, LP, M. Krssak, and M. Krebs analyzed data and wrote the manuscript. SW, AM, WR, TS, ST, and AKW contributed to the study design and manuscript preparation. All authors reviewed the manuscript and provided final approval for submission.

\section{Acknowledgments}

This study was supported by the anniversary fund of the Oesterreichische Nationalbank (no. 16724 to YW) and an unrestricted grant from Ipsen Pharma (to M. Krebs).

Address correspondence to: Yvonne Winhofer, Department for Internal Medicine III, Division of Endocrinology and Metabolism, Währinger Gürtel 18-20; 1090 Vienna, Austria. Phone: 0043.1.40400.43120; Email: yvonne.winhofer@meduniwien.ac.at

1. Winhofer Y, et al. No evidence of ectopic lipid accumulation in the pathophysiology of the acromegalic cardiomyopathy. JClin Endocrinol Metab. 2014;99(11):4299-4306.

2. Bredella MA, et al. Body composition and ectopic lipid changes with biochemical control of acromegaly. J Clin Endocrinol Metab. 2017;102(11):4218-4225.

3. Vila G, Jørgensen JOL, Luger A, Stalla GK. Insulin resistance in patients with acromegaly. Front Endocrinol (Lausanne). 2019;10:509.

4. Hansen I, Tsalikian E, Beaufrere B, Gerich J, Haymond M, Rizza R. Insulin resistance in acromegaly: defects in both hepatic and extrahepatic insulin action. Am J Physiol. 1986;250(3 Pt 1):E269-E273.

5. Michelotti GA, Machado MV, Diehl AM. NAFLD, NASH and liver cancer. Nat Rev Gastroenterol Hepatol. 2013;10(11):656-665.

6. Petersen KF, Dufour S, Befroy D, Garcia R, Shulman GI. Impaired mitochondrial activity in the insulin-resistant offspring of patients with type 2 diabetes. $N$ Engl J Med. 2004;350(7):664-671.

7. Nishizawa H, Takahashi M, Fukuoka H, Iguchi G, Kitazawa R, Takahashi Y. GH-independent IGF-I action is essential to prevent the development of nonalcoholic steatohepatitis in a GH-deficient rat model. Biochem Biophys Res Commun. 2012;423(2):295-300.

8. Liu Z, et al. Growth hormone control of hepatic lipid metabolism. Diabetes. 2016;65(12):3598-3609.

9. Takahashi Y. The role of growth hormone and insulin-like growth factor-I in the liver. Int J Mol Sci. 2017;18(7):E1447.

10. Short KR, Moller N, Bigelow ML, Coenen-Schimke J, Nair KS. Enhancement of muscle mitochondrial function by growth hormone. J Clin Endocrinol Metab. 2008;93(2):597-604.

11. Stanley TL, et al. Effects of tesamorelin on non-alcoholic fatty liver disease in HIV: a randomised, double-blind, multicentre trial. Lancet HIV. 2019;6(12):e821-e830.

12. Anderwald C, et al. Effects of insulin treatment in type 2 diabetic patients on intracellular lipid content in liver and skeletal muscle. Diabetes. 2002;51(10):3025-3032.

13. Phielix E, et al. Effects of pioglitazone versus glimepiride exposure on hepatocellular fat content in type 2 diabetes. Diabetes Obes Metab. 2013;15(10):915-922.

14. Krssak M, et al. Alterations in postprandial hepatic glycogen metabolism in type 2 diabetes. Diabetes. 2004;53(12):3048-3056.

15. Koliaki C, Roden M. Hepatic energy metabolism in human diabetes mellitus, obesity and non-alcoholic fatty liver disease. Mol. Cell. Endocrinol. 2013;379(1):35-42.

16. Møller N, et al. Basal- and insulin-stimulated substrate metabolism in patients with active acromegaly before and after adenomectomy. J Clin Endocrinol Metab. 1992;74(5):1012-1019.

17. Sjögren K, Leung KC, Kaplan W, Gardiner-Garden M, Gibney J, Ho KK. Growth hormone regulation of metabolic gene expression in muscle: a microarray study in hypopituitary men. Am J Physiol Endocrinol Metab. 2007;293(1):E364-E371.

18. Friedbichler K, et al. Growth-hormone-induced signal transducer and activator of transcription 5 signaling causes gigantism, inflammation, and premature death but protects mice from aggressive liver cancer. Hepatology. 2012;55(3):941-952.

19. Miquet JG, et al. Hepatocellular alterations and dysregulation of oncogenic pathways in the liver of transgenic mice overexpressing growth hormone. Cell Cycle. 2013;12(7):1042-1057.

20. Valkovič L, et al. Application of localized ${ }^{31} \mathrm{P}$ MRS saturation transfer at $7 \mathrm{~T}$ for measurement of ATP metabolism in the liver: reproducibility and initial clinical application in patients with non-alcoholic fatty liver disease. Eur Radiol. 2014;24(7):1602-1609.

21. Schmid AI, et al. Quantitative ATP synthesis in human liver measured by localized 31P spectroscopy using the magnetization transfer experiment. NMR Biomed. 2008;21(5):437-443.

22. Ribel-Madsen A, Ribel-Madsen R, Brøns C, Newgard CB, Vaag AA, Hellgren LI. Plasma acylcarnitine profiling indicates 
increased fatty acid oxidation relative to tricarboxylic acid cycle capacity in young, healthy low birth weight men. Physiol Rep . 2016;4(19):e12977.

23. Loomba R, et al. GS-0976 Reduces hepatic steatosis and fibrosis markers in patients with nonalcoholic fatty liver disease. Gastroenterology. 2018;155(5):1463-1473.e6.

24. Mardinoglu A, et al. Personal model-assisted identification of $\mathrm{NAD}^{+}$and glutathione metabolism as intervention target in NAFLD. Mol Syst Biol. 2017;13(3):916.

25. Gaggini M, et al. Altered amino acid concentrations in NAFLD: Impact of obesity and insulin resistance. Hepatology. 2018;67(1):145-158.

26. Gajdošík M, et al. Ultrashort-TE stimulated echo acquisition mode (STEAM) improves the quantification of lipids and fatty acid chain unsaturation in the human liver at 7T. NMR Biomed. 2015;28(10):1283-1293.

27. Puri P, et al. A lipidomic analysis of nonalcoholic fatty liver disease. Hepatology. 2007;46(4):1081-1090.

28. Johnson NA, et al. Noninvasive assessment of hepatic lipid composition: Advancing understanding and management of fatty liver disorders. Hepatology. 2008;47(5):1513-1523.

29. Traussnigg S, et al. Ultra-high-field magnetic resonance spectroscopy in non-alcoholic fatty liver disease: Novel mechanistic and diagnostic insights of energy metabolism in non-alcoholic steatohepatitis and advanced fibrosis. Liver Int. 2017;37(10):1544-1553

30. Khan SA, Cox IJ, Hamilton G, Thomas HC, Taylor-Robinson SD. In vivo and in vitro nuclear magnetic resonance spectroscopy as a tool for investigating hepatobiliary disease: a review of $\mathrm{H}$ and P MRS applications. Liver Int. 2005;25(2):273-281.

31. Solga SF, Horska A, Clark JM, Diehl AM. Hepatic 31P magnetic resonance spectroscopy: a hepatologist's user guide. Liver Int. 2005;25(3):490-500.

32. Olarescu NC, Bollerslev J. The impact of adipose tissue on insulin resistance in acromegaly. Trends Endocrinol Metab. 2016;27(4):226-237.

33. Corbit KC, et al. Adipocyte JAK2 mediates growth hormone-induced hepatic insulin resistance. JCI Insight. 2017;2(3):e91001.

34. Foss MC, Saad MJ, Paccola GM, Paula FJ, Piccinato CE, Moreira AC. Peripheral glucose metabolism in acromegaly. J Clin Endocrinol Metab. 1991;72(5):1048-1053.

35. Higham CE, Rowles S, Russell-Jones D, Umpleby AM, Trainer PJ. Pegvisomant improves insulin sensitivity and reduces overnight free fatty acid concentrations in patients with acromegaly. J Clin Endocrinol Metab. 2009;94(7):2459-2463.

36. Petersen MC, Shulman GI. Mechanisms of insulin action and insulin resistance. Physiol Rev. 2018;98(4):2133-2223.

37. Krssak M, Roden M. The role of lipid accumulation in liver and muscle for insulin resistance and type 2 diabetes mellitus in humans. Rev Endocr Metab Disord. 2004;5(2):127-134.

38. Krssak M, et al. Intramyocellular lipid concentrations are correlated with insulin sensitivity in humans: a 1H NMR spectroscopy study. Diabetologia. 1999;42(1):113-116.

39. Reyes-Vidal CM, et al. Adipose tissue redistribution and ectopic lipid deposition in active acromegaly and effects of surgical treatment. J Clin Endocrinol Metab. 2015;100(8):2946-2955.

40. Kemp GJ, Brindle KM. What do magnetic resonance-based measurements of Pi $\rightarrow$ ATP flux tell us about skeletal muscle metabolism? Diabetes. 2012;61(8):1927-1934.

41. Szendroedi J, et al. Muscle mitochondrial ATP synthesis and glucose transport/phosphorylation in type 2 diabetes. PLoS Med. 2007;4(5):e154

42. Pfleger L, et al. Absolute Quantification of phosphor-containing metabolites in the liver using ${ }^{31} \mathrm{P}$ MRSI and hepatic lipid volume correction at 7T suggests no dependence on body mass index or age. J Magn Reson Imaging. 2019;49(2):597-607.

43. Klepochová R, et al. Differences in muscle metabolism between triathletes and normally active volunteers investigated using multinuclear magnetic resonance spectroscopy at 7T. Front Physiol. 2018;9:300.

44. Wallace TM, Levy JC, Matthews DR. Use and abuse of HOMA modeling. Diabetes Care. 2004;27(6):1487-1495.

45. Mari A, Pacini G, Murphy E, Ludvik B, Nolan JJ. A model-based method for assessing insulin sensitivity from the oral glucose tolerance test. Diabetes Care. 2001;24(3):539-548.

46. Matsuda M, DeFronzo RA. Insulin sensitivity indices obtained from oral glucose tolerance testing: comparison with the euglycemic insulin clamp. Diabetes Care. 1999;22(9):1462-1470.

47. Goyal G, et al. Sphingolipid-dependent Dscam sorting regulates axon segregation. Nat Commun. 2019;10(1):813.

48. Chong J, Wishart DS, Xia J. Using MetaboAnalyst 4.0 for Comprehensive and integrative metabolomics data analysis. Curr Protoc Bioinformatics. 2019;68(1):e86. 